\title{
Construction of analytical eigensolutions for isotropic conical bodies and their application for estimation of stresses singularity
}

\author{
V. Matveenko, I. Shardakov \\ Institute of Continuous Media Mechanics of the Ural Branch of Russian Academy of Sciences, Russia \\ mvp@icmm.ru \\ shardakov@icmm.ru \\ T. Korepanova \\ Institute of Continuous Media Mechanics of the Ural Branch of Russian Academy of Sciences, Russia \\ Perm Military Institute of the National Guard Troops of the Russian Federation, Russia \\ ton@icmm.ru
}

\begin{abstract}
A complete set of eigensolutions is constructed for different variants of circular conical bodies: homogeneous cone with one lateral surface (solid cone), homogeneous cone with two lateral surfaces (hollow cone) and composite cone for different boundary conditions on the lateral surface. It has been shown that the constructed eigensolutions can be readily applied for estimation of the character of stress singularity at the vertices of conical bodies. The character of stress singularity at the vertex of the solid and hollow cones for different boundary conditions on the lateral surfaces has been defined by direct numerical simulations. Numerical results obtained for solid, hollow and compose cones under different boundary conditions on the lateral surfaces are discussed.
\end{abstract}

KEYWORDS. Stress singularity; Conical bodies; Eigensolutions.

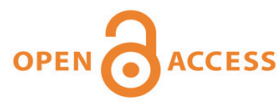

Citation: Matveenko, V., Shardakov, I., Korepanova, T., Construction of analytical eigensolutions for isotropic conical bodies and their application for estimation of stresses singularity , Frattura ed Integrità Strutturale, 49 (2019) 225-242

Received: 17.04 .2019

Accepted: 24.05 .2019

Published: 01.07.2019

Copyright: (C) 2019 This is an open access article under the terms of the CC-BY 4.0, which permits unrestricted use, distribution, and reproduction in any medium, provided the original author and source are credited.

\section{INTRODUCTION}

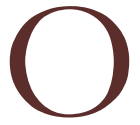
ne of the main findings of the classical elasticity theory is the possibility of existence of singular solutions due to the occurrence of infinite stresses at the points of surface non-smoothness, changes in the type of boundary conditions, contact of different materials and inside the body at the points of contact of dissimilar materials. An example of theoretical justification of this statement can be found in Kondratiev (1967), where it was shown that the solutions to the equations of the linear theory of elasticity in the vicinity of angular points can be expressed as 


$$
\tau \sim \sum_{\mathrm{n}=1}^{\infty} \mathrm{f}_{\mathrm{n}} \mathrm{r}^{\alpha_{\mathrm{n}}-1}, \mathrm{r} \rightarrow 0, \mathrm{c}<\operatorname{Re} \alpha_{1}<\operatorname{Re} \alpha_{2}<\ldots<\operatorname{Re} \alpha_{\mathrm{n}}<\ldots
$$

or can be written in a more complicated form involving logarithmic components in the case of multiple spectrum points $\alpha_{n}$. Here $r$ is the distance to the angular point, $f_{n}$ is the function of an angular distribution of the stress field $\tau$ in the vicinity of an angular point, which in $2 \mathrm{D}$ case depends on one polar angular variable $\varphi, c=0$, and in $3 \mathrm{D}$ case - on two spherical coordinates $\varphi, \theta, c=-1 / 2$.

Such a representation of the problem solution suggests that if there are $\alpha_{n}$ with $\operatorname{Re} \alpha_{n}<1$, the stresses tend to infinity at $r$ tends to zero. One of the trends in the construction of the solutions of form (1) concerns the analysis of regions with specific configuration. In two-dimensional problems these are plane wedges. For almost half-a century history of studying these problems the researchers have investigated nearly all types of wedge-shaped bodies: homogeneous, composite, isotropic, anisotropic, etc. In [2-3] the authors give an extended review of the literature concerning the construction and analysis of singular solutions in two-dimensional problems of the theory of elasticity.

In three-dimensional problems one can differentiate between two types of regions: an edge of a 3D wedge (an edge is not necessarily rectilinear, the wedge angle can vary along the edge) and a vertex of a polyhedral wedge or a cone. The results of some publications have disengaged further interest in the problems of the first class. In these articles (for example, in [4]) it has been shown that the solution of the plane and anti-plane problems for wedges obtained in the planes perpendicular to the edge of a 3D wedge defines the type of stress singularity at the edge points, through which the plane passes.

In recent years, considerable attention has been focused on studying the stress singularity at the vertices of polyhedral wedges or cones. These problems are solved by different algorithms of the finite element method, the boundary elements method or by applying the Mellin transformation to the two-dimensional boundary integral equations. Out of numerous papers, which have used the concepts of these numerical methods, one should set aside papers [5-12].

As in the other branches of the elasticity theory, the analytical methods are of considerable importance both for numerical simulation and testing of numerical methods. In three-dimensional problems the analytical methods are generally applied to circular cones. One of the pioneering works in this field Bazant and Keer [13] is concerned with the stress singularity problem for a solid cone under axisymmetric deformation and torsion, for which the boundary conditions are specified in terms of displacement and stresses. A detailed theoretical analysis of the construction of axisymmetric solutions for elastic cone is given in [14]. The method of construction of singular and regular solutions to the Laplace and Navier-Stokes equations written for the axisymmetric domain is described in [15]. The idea of the method is the expansion of the desired solution in the Fourier series in the cyclic coordinate for axisymmetric coordinate systems, which is followed by the numerical solution of a sequence of two-dimensional problems for series coefficients.

In the papers that followed the above cited studies, the analytical solutions were constructed for some particular problems. For example, in [16-17] the authors presented the results for a composite cone under axisymmetric deformation. In this case, a composite cone is a body composed of two embedded cones, which have a common contact surface. The solutions were obtained for the perfect bonding and frictionless slip conditions. The axisymmetric problem for a circular cone made of transversally isotropic material was considered in [18]. A rather complete list of papers dealing with the investigation of circular cones by the analytical methods can be found in review article [3].

The most comprehensive research on this topic was carried out in [19], in which the authors constructed the analytical solution for a solid circular cone and presented numerical data on the character of stress singularity at the vertex of a circular solid cone with the boundary conditions at the lateral surface specified in terms of the displacements and stresses. Compared to the known results, the present work presents a full spectrum of analytical eigensolutions, which allow constructing the solutions not only for a homogeneous isotropic cone but also for other types of isotropic cone, as for example, for hollow and composite cones for different boundary conditions on the lateral surfaces.

\section{MATHEMATICAL STATEMENT OF THE PROBLEM OF CONSTRUCTING EIGENSOLUTIONS FOR SEMI- INFINITE CIRCULARCONICAL BODIES}

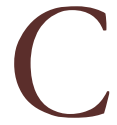

onsider a homogeneous circular cone (Fig. 1a), the vertex of which coincides with the center of spherical coordinates $r, \theta, \varphi$, and the base is perpendicular to the axis $\theta=0$. The volume occupied by the cone is defined as $0 \leq r<\infty, \theta_{1} \leq \theta \leq \theta_{0}, 0 \leq \varphi \leq 2 \pi$, and its boundary is defined by the coordinate surfaces $\theta=\theta_{1}, \theta=\theta_{0}$. 
The case when $\theta_{1}=0$ corresponds to a solid cone.
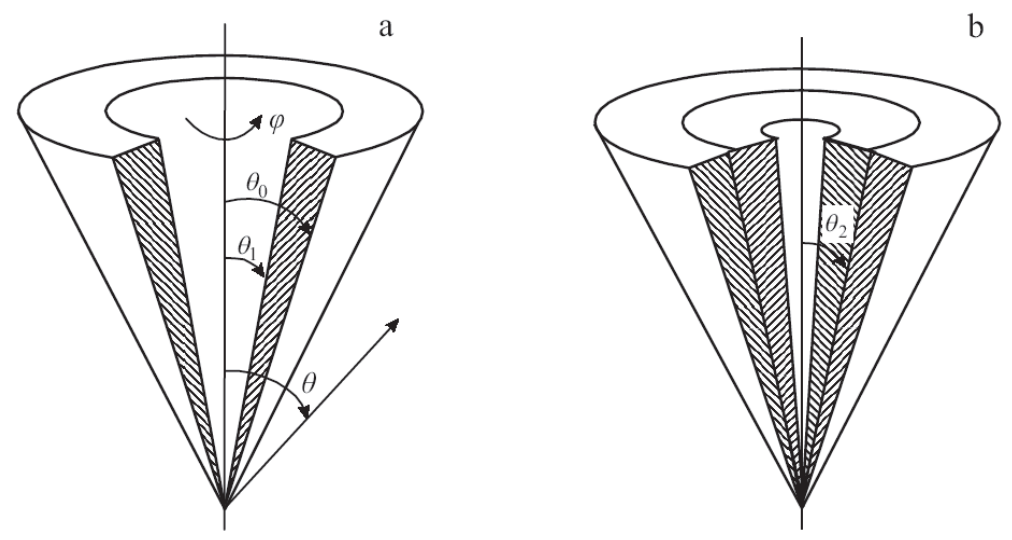

Figure 1: Hollow cone (a); composite cone (b).

We state the problem of constructing eigensolutions, which will satisfy the homogeneous equations of equilibrium

$$
(1+S) \operatorname{grad} \operatorname{div} \mathbf{u}-\operatorname{rot} \operatorname{rot} \mathbf{u}=0
$$

(here $S=1 / 1-2 \nu, \nu$ is Poisson's ratio, $\mathbf{u}$ is the displacement vector)

and homogeneous boundary conditions at the surfaces $\theta=\theta_{1}, \theta=\theta_{0}$ for displacements

$$
u_{r}=0, u_{\theta}=0, u_{\varphi}=0
$$

and stresses

$$
\sigma_{r \theta}=0, \sigma_{\theta \theta}=0, \sigma_{\theta \varphi}=0
$$

or mixed boundary conditions, which in the context of solid mechanics correspond to a perfectslip boundary condition at the lateral surface

$$
u_{\theta}=0, \sigma_{r \theta}=0, \sigma_{\theta \varphi}=0
$$

For the examined body of revolution and boundary conditions (3)-(5), the eigensolutions can be represented as a Fourier series [20] in the circumferential coordinate $\varphi$

$$
\begin{aligned}
& u_{r}(r, \theta, \varphi)=u_{0}(\theta) r^{\alpha}+\sum_{k=1}^{\infty}\left[u_{k}(\theta) r^{\alpha} \sin (k \varphi)\right] \\
& u_{\theta}(r, \theta, \varphi)=\mathrm{v}_{0}(\theta) r^{\alpha}+\sum_{k=1}^{\infty}\left[\mathrm{v}_{k}(\theta) r^{\alpha} \sin (k \varphi)\right] \\
& u_{\varphi}(r, \theta, \varphi)=w_{0}(\theta) r^{\alpha}+\sum_{k=1}^{\infty}\left[w_{k}(\theta) r^{\alpha} \cos (k \varphi)\right]
\end{aligned}
$$

Here the dependence on the radius is expressed according to (1), $u_{r}, u_{\theta}, u_{\varphi}$-are the components of the displacement vector along the $r, \theta, \varphi$-axes, $\sigma_{r \theta}, \sigma_{\theta \theta}, \sigma_{\theta \varphi}$ are the components of the stress tensor, $\alpha$ is the characteristic index. 
If $\theta_{1}=0$, the region under consideration is bounded by only one coordinate surface $\theta=\theta_{0}$, and at $\theta=0$ is assumed to meet the regularity conditions

$$
\partial u_{r} / \partial \theta=0, u_{\theta}=0, u_{\varphi}=0
$$

In the framework of the proposed problem formulation we can consider a composite cone occupying a region $V=V^{(1)}+V^{(2)}$, where a subregion $V^{(1)}$ (subregion $\left.V^{(2)}\right)$ is made of the material with the shear modulus $\mu^{(1)}\left(\mu^{(2)}\right)$ and Poisson's ratio $v^{(1)}\left(v^{(2)}\right)$ and its geometry is defined by the relations $0 \leq r \leq \infty, 0 \leq \varphi \leq 2 \pi, \theta_{2} \leq \theta \leq \theta_{0}$ $\left(\theta_{1} \leq \theta \leq \theta_{2}\right)$ (Fig. 1b). In particular cases $\theta_{1}$ and $\theta_{0}$ can be equal to 0 and $\pi$, respectively. For a composite cone eigensolutions (6) are constructed for each subregion. At the contact line $\theta=\theta_{2}$ we can prescribe perfect bonding conditions

$$
u_{r}^{(1)}=u_{r}^{(2)}, u_{\varphi}^{(1)}=u_{\varphi}^{(2)}, u_{\theta}^{(1)}=u_{\theta}^{(2)} ; \sigma_{\theta}^{(1)}=\sigma_{\theta}^{(2)}, \tau_{r \theta}^{(1)}=\tau_{r \theta}^{(2)}, \tau_{\varphi \theta}^{(1)}=\tau_{\varphi \theta}^{(2)}
$$

or perfect slip conditions

$$
u_{\theta}^{(1)}=u_{\theta}^{(2)} ; \sigma_{\theta}^{(1)}=\sigma_{\theta}^{(2)}, \tau_{r \theta}^{(1)}=\tau_{r \theta}^{(2)}=\tau_{\varphi \theta}^{(1)}=\tau_{\varphi \theta}^{(2)}=0
$$

Upon substituting Eqns. (6) into the equilibrium Eqn. (2) and going to a new independent variable $x=(1-\cos \theta) / 2$ [20] we obtain the following equations for each harmonic of the Fourier series:

$$
\begin{aligned}
& (x)(1-x) \frac{d^{2} u_{k}(x)}{d x^{2}}+(1-2 x) \frac{d u_{k}(x)}{d x}+\frac{\left[4 x H_{1}(x-1)+k^{2}\right]}{4 x(x-1)} u_{k}(x)+ \\
& +\frac{x(1-x) H_{2}}{\sqrt{x(1-x)}} \frac{d v_{k}(x)}{d x}+\frac{H_{2}}{\sqrt{x(1-x)}}\left[\left(\frac{1}{2}-x\right) \mathrm{v}_{k}(x)-\frac{k w_{k}(x)}{2}\right]=0 \\
& G_{1} x(1-x) \frac{d^{2} v_{k}(x)}{d x^{2}}+G_{1}(1-2 x) \frac{d v_{k}(x)}{d x}+\frac{\left[4 x G_{2}(x-1)+k^{2}+G_{1}\right]}{4 x(x-1)} v_{k}(x)+ \\
& +G_{3} \sqrt{x(1-x)} \frac{d}{d x} u_{k}(x)+\left[\frac{k\left(1-G_{1}\right)}{2} \frac{d w_{k}(x)}{d x}+\frac{\left(G_{1}+1\right) k(2 x-1)}{4 x(x-1)} w_{k}(x)\right]=0 \\
& (x)(1-x) \frac{d^{2} w_{k}}{d x^{2}}+(x) \\
& +\frac{k G_{3}}{2 \sqrt{x(1-x)}} u_{k}(x)+\left[\frac{\left(G_{1}-1\right) k}{2} \frac{d v_{k}(x)}{d x}+\frac{\left(G_{1}+1\right) k(2 x-1)}{4 x(x-1)} \cdot v_{k}(x)\right]=0 \\
& w_{k}(x)+
\end{aligned}
$$

Here we introduce the following notation:

$$
\begin{aligned}
& H_{1}=2(1-v)(1-\alpha)(\alpha+2) /(2 v-1), H_{2}=(3-\alpha-4 v) /(2 v-1), \\
& G_{1}=2(1-v) /(1-2 v), G_{2}=\alpha(1+\alpha), G_{3}=2(\alpha+4-4 v) /(1-2 v) .
\end{aligned}
$$

Using (6) we can reduce boundary conditions (3)-(5) and regularity conditions (7) to condition for 
displacements

$$
u_{k}(x)=0, \quad v_{k}(x)=0, \quad w_{k}(x)=0
$$

stresses

$$
\begin{aligned}
& \mu\left[\sqrt{x(1-x)} \frac{d u_{k}(x)}{d x}+(\alpha-1) v_{k}(x)\right]=0 \\
& \mu\left[(2 S-\alpha+\alpha S) u_{k}(x)+(1+S) \sqrt{x(1-x)} \frac{d v_{k}(x)}{d x_{j}}+\right. \\
& \left.+\left(\frac{1}{2}-x\right) \frac{(S-1)}{\sqrt{x(1-x)}} v_{k}(x)+\frac{k(1-S)}{2 \sqrt{x(1-x)}} w_{k}(x)\right]=0 \\
& \mu\left[\sqrt{x(1-x)} \frac{d w_{k}(x)}{d x}-\frac{(1-2 x)}{2 \sqrt{x(1-x)}} w_{k}(x)+\frac{k}{2 \sqrt{x(1-x)}} v_{k}(x)\right]=0 \\
& \sqrt{x(1-x)} \frac{d u_{k}(x)}{d x}=0 ; \quad v_{k}(x)=0 ; \quad w_{k}(x)=0 .
\end{aligned}
$$

\section{CONSTRUCTION OF PARTIAL SOLUTION FOR ZEROTH HARMONIC OF THE FOURIER SERIES}

A solution for zeroth harmonic of the Fourier series is considered separately, because it does not follow explicitly from the algorithm for construction of partial solutions to the system of differential Eqns. (10)-(12) for any $k \neq 0$. At $k=0$ there are two problems: axisymmetric rotation and axisymmetric deformation. In the first problem, the component of the displacement vector $w_{0}$ is defined by Eqn. (12). In the problem of axisymmetric deformation the components of the displacement vector $u_{0}, v_{0}$ are defined by Eqns. (10), (11).

The solutions for the function $w_{0}$ are constructed in the form of the generalized power series

$$
w_{0}(x)=\sum_{m=0}^{\infty}\left[R_{m} x^{(m+\beta)}\right]
$$

where $R_{m}$ are the coefficients of the series, $\beta$ is the characteristic index.

The possibility of constructing a solution in the form of (16) was proved in [21]. For Eqn. (12) the point $x=0$ is a regular singular point. In this case, one of the partial solutions is written as a power series (16), for which the range of convergence lies in the interval $0 \leq x \leq 1$, because the value $x=1$ is the zero of the high-order derivative. function closest to the point $x=0$.

The coefficients of the series $R_{m}$ and the characteristic index $\beta$ are found by substituting (16) into (12). Setting to zero the expressions with like powers of $x$, yields a recurrence relation for $R_{m}$ :

$$
\begin{aligned}
& (2 \beta+2 m+1)(2 \beta+2 m-1) \mathrm{R}_{m}+4[\alpha(\alpha+1)-(2 \beta+2 m-1)(\beta+m-1)] \mathrm{R}_{m-1}- \\
& -4(\alpha+2-m-\beta)(\alpha-1+m+\beta) \mathrm{R}_{m-2}=0,\left(\mathrm{R}_{m}=0, \text { if } m<0\right)
\end{aligned}
$$

From the condition of existence of non-zero solution for $R_{0}$ we obtain the characteristic equation:

$$
(2 \beta+1)(2 \beta-1)=0
$$

for which $\beta_{1}=1 / 2$ and $\beta_{2}=-1 / 2$ are the roots. 
According to the theory of differential equations [21], there always exists a solution in the form of a generalized power series (16), that fits the largest root $\beta_{1}$. Substituting the value of the root $\beta_{1}$ into (17), we obtain a recurrence relation for $\mathrm{R}_{m}^{(1)}$

$$
R_{m}^{(1)}=\frac{\left(2 m^{2}-\alpha-\alpha^{2}-m\right)}{m(1+m)} R_{m-1}^{(1)}+\frac{(2 \alpha-1+2 m)(2 \alpha+3-2 m)}{4 m(1+m)} R_{m-2}^{(1)}, \quad\left(m>0, R_{0}^{(1)}=1\right)
$$

Here in after, the superscript will denote the number of the partial solution.

After completing these transformations we obtain the first partial solution for Eqn. (12) represented as a generalized power series

$$
w_{0}^{(1)}(x)=\sum_{m=0}^{\infty}\left[R_{m}^{(1)} x^{\left(m+\frac{1}{2}\right)}\right]
$$

A quantity that plays an important part in the construction of the second, linearly independent partial solution in the form of the generalized power series is the difference in the roots of the characteristic equation [21], i.e. the number $\gamma=\beta_{1}-\beta_{2}=1$. If the number $\gamma$ is not a positive integer there exists a second, linearly independent solution in the form of the generalized power series (16). However, if $\gamma$ is a positive integer, the existence of the second partial solution in the general case in the form of a generalized power series (16) is not assured. This is exactly the case we have, because $\gamma=\beta_{1}-\beta_{2}=1$.

To eliminate this ambiguity, we use an approach, which is based on a subsequent reduction of the initial differential equation and retention of a certain number of series terms.

The performance of this technique is illustrated by way of constructing the second particular solution $w_{0}^{(2)}(x)$ to Eqn. (12) at $k=0$, which corresponds to axisymmetric deformation.

Eqn. (12) at $k=0$ takes the following form:

$$
(x)(1-x) \frac{d^{2} w_{0}(x)}{d x^{2}}+(1-2 x) \frac{d w_{0}(x)}{d x}+\frac{\left[4 x G_{2}(x-1)+G_{1} k^{2}+1\right]}{4 x(x-1)} w_{0}(x)=0
$$

The construction of the second particular solution proceeds as follows. At the first stage using the obtained first particular solution $w_{0}^{(1)}(x)$ (20) we reduce the second order differential Eqns. (21) to the first order equation replacing the desired solution in Eqn. (21) by the relation [21]

$$
w_{0}(x)=w_{0}^{(1)}(x) \cdot \int F(x) d x
$$

where $F(x)$ is a new unknown function.

Upon this substitution we obtain the linear first-order differential equation for

$$
P_{1}(x) \frac{d F(x)}{d x}+P_{0}(x) F(x)=0
$$

where

$$
\begin{aligned}
& P_{1}(x)=x(1-x) w_{0}^{(1)}(x) \\
& P_{0}(x)=(1-x) w_{0}^{(1)}(x)+2\left(x-x^{2}\right) \frac{d w_{0}^{(1)}(x)}{d x} .
\end{aligned}
$$


For this equation we can also construct a solution [21] for $\mathrm{F}(\mathrm{x})$ in the form of a generalized power series

$$
F(x)=\sum_{m=0}^{\infty}\left[N_{m} x^{(m+\xi)}\right]
$$

In the traditional approach, the coefficients $N_{m}$ and index $\xi$ of this series are determined by substituting (25) into Eqn. (23). By setting the expressions of the same power of $\chi$ equal to zero we can obtain the recurrence relations for $N_{m}$ and from the condition of the existence of zero solution for $N_{0}$ we get the characteristic equation, determining the index $\gamma$. However, it should be noted that practical implementation of this procedure presents considerable difficulties, because the expressions in front of the function $F(x)$ and its derivatives in Eqn. (23) will appear in the form of in finite series. Moreover, the fact that that after construction of series (25) it will be necessary to proceed to the solution $w_{0}(x)$ through the relation (22), implies that analytical transformations of infinite series will become increasingly complex.

In this regard, we suggest using an algorithm, which allows us to circumvent the above difficulties in the construction of a particular solution. To illustrate our ides we will apply the proposed algorithm to Eqn. (23).

1. At the first stage we define the form of the characteristic equation for the index $\xi$. For this purpose, we retain in the series (25) only the first term with the coefficient $N_{0}$, and in the expressions (24) in front of the function $F(x)$ and its derivatives in Eqn. (23) we retain only the lowest powers of the variable $x$, which, however are not less than the order of the highest derivative (in this case, for Eqn. (23) this is the first order). After these manipulations the coefficients (24) take the following form:

$$
\begin{aligned}
& P_{1}(x)=x(1-x) R_{0}^{(1)} x^{1 / 2} \\
& P_{0}(x)=(1-2 x)\left(R_{0}^{(1)} x^{1 / 2}+R_{1}^{(1)} x^{3 / 2}\right)+2\left(x-x^{2}\right)\left(1 / 2 R_{0}^{(1)} x^{-1 / 2}+3 / 2 R_{1}^{(1)} x^{1 / 2}\right) .
\end{aligned}
$$

2. Upon substituting relations (25) and (26), in which we retain only the first term with the coefficient $N_{0}$, into Eqn. (23) and setting to zero the expression with the lowest power of $x$, we obtain

$$
N_{0}(\xi+2)=0
$$

From (27) it follows that the characteristic index $\xi=-2$. With a knowledge of this index we can formally represent the structure of the solution for $F(x)$ :

$$
F(x)=N_{0} x^{-2}+N_{1} x^{-1}+N_{2} x^{0}+N_{3} x+\ldots
$$

Using the obtained solution (28), we can find a formal representation of the second particular solution for $w_{0}(x)$ based on the relation (22). In this case the number of the series terms retained in the series (28) and (20) should be no less than $|\xi|$ (namely, no less than 2). Substitution of these segments of the series into relation (22) yields

$$
w_{0}^{(1)}(x) \cdot \int F(x) d x=\sum_{m=0}^{3}\left\{\left[R_{m}^{(2)} x+T_{m}^{(2)} \cdot \ln (x)\right] x^{(1 / 2)}\right\}
$$

Thus, after generalization of this form of relation to the infinite number of terms we obtain the following form of the second particular solution:

$$
\omega_{0}^{(2)}(x)=\sum_{m=0}^{\infty}\left\{\left[R_{m}^{(2)}+T_{m}^{(2)} \cdot \ln (x)\right] \cdot x^{(1 / 2)}\right\}
$$

In this expression the coefficients $R_{m}^{(2)}, T_{m}^{(2)}$ are still to be defined. 
3. Substituting relation (30) into the initial differential Eqn. (21) and equating to zero the expressions with the same powers of $x$, we obtain the recurrence relations for determination of the coefficients $R_{m}^{(2)}, T_{m}^{(2)}$

$$
\begin{aligned}
& T_{m}^{(2)}=\frac{\left[(m-1)(2 m-3)-\alpha^{2}-\alpha\right]}{m(m-1)} T_{m-1}^{(2)}-\frac{(2 m-3+2 \alpha)(2 m-5-2 \alpha)}{4 m(m-1)} T_{m-2}^{(2)} \\
& R_{m}^{(2)}=\frac{(1-2 m)}{m(m-1)} T_{m}^{2}+\frac{\left[(m-1)(2 m-3)-\alpha^{2}-\alpha\right]}{m(m-1)} R_{m-1}^{(2)}+\frac{(4 m-5)}{m(m-1)} T_{m-1}^{(2)}- \\
& -\frac{(2 m-3+2 \alpha)(2 m-5-2 \alpha)}{4 m(m-1)} R_{m-2}^{(2)}-\frac{2(m-2)}{m(m-1)} T_{m-2}^{(2)} \\
& \left(m>1, \quad T_{0}^{(2)}=1, \quad T_{1}^{(2)}=-\alpha(\alpha+1), \quad R_{0}^{(2)}=1, \quad R_{1}^{(2)}=1\right)
\end{aligned}
$$

The proposed algorithm is valid for the linear ordinary differential equations of any order.

Hence, the proposed method allows us to define step by step the types of the generalized power series for all partial solutions of the initial differential equation and in all partial solutions to set apart the regular from irregular solutions (in our case for $x=0$ ). Such a capability of the method is rather essential for constructing solutions to particular problems, for example, for hollow and composite cones.

The form of the obtained solutions $w_{0}^{(1)}, w_{0}^{(2)}$ suggests that $w_{0}^{(1)}$ is a regular solution, and $w_{0}^{(2)}$ is irregular solution at $x=0$.

A general solution to differential Eqn. (12) takes the following form:

$$
w_{0}(x)=C_{1} \cdot w_{0}^{(1)}(x)+C_{2} \cdot w_{0}^{(2)}(x)
$$

where $C_{1}, C_{2}$ are the constants defined by the prescribed combination of the boundary conditions (3) - (5).

To construct partial solutions to Eqns. (10), (11), corresponding to the axisymmetric deformation, we need to solve this system for $v_{0}$

$$
v_{0}(x)=\frac{\sqrt{x(1-x)}}{(1-\alpha) S+2}\left\{\frac{(S+1)}{\left(\alpha+\alpha^{2}\right)}\left[\left(x^{2}-x\right) \frac{d^{3} u_{0}(x)}{d x^{3}}+(4 x-2) \frac{d^{2} u_{0}(x)}{d x^{2}}\right]-(2 S+1) \frac{d u_{0}(x)}{d x}\right\}
$$

and with respect to the function $u_{0}$, which yields a differential equation of the fourth order

$$
\begin{aligned}
& x^{2}(x-1)^{2} \frac{d^{4} u_{0}(x)}{d x^{4}}-x(x-1)(4-8 x) \frac{d^{3} u_{0}(x)}{d x^{3}}+[2-2 x(\alpha+3)(\alpha-2)(-1+x)] \frac{d^{2} u_{0}(x)}{d x^{2}}- \\
& -\alpha(2+2 \alpha)(2 x-1) \frac{d u_{0}(x)}{d x}-\left(\alpha+\alpha^{2}\right)(1-\alpha)(2+\alpha) u_{0}(x)=0
\end{aligned}
$$

This equation is the differential equation with a regular singular point. Therefore, the linearly independent partial solutions can be represented in the form of convergent generalized power series. Using the above procedure for constructing such series, we obtain four partial solutions $u_{0}^{(1)}, u_{0}^{(2)}, u_{0}^{(3)}, u_{0}^{(4)}$ written as

$$
\begin{array}{ll}
u_{0}^{(1)}(x)=\sum_{m=0}^{\infty}\left[A_{m}^{(1)} x^{(m+1)}\right], & u_{0}^{(2)}(x)=\sum_{m=0}^{\infty}\left[A_{m}^{(2)} x^{m}\right] \\
u_{0}^{(3)}(x)=\sum_{m=0}^{\infty}\left\{\left[A_{m}^{(3)}+B_{m}^{(3)} \ln (x)\right] x^{(m+1)}\right\}, & u_{0}^{(4)}(x)=\sum_{m=0}^{\infty}\left\{\left[A_{m}^{(4)}+B_{m}^{(4)} \ln (x)\right] x^{m}\right\},
\end{array}
$$


where the coefficients $A_{m}^{(1)}, A_{m}^{(2)}, A_{m}^{(3)}, A_{m}^{(4)}, B_{m}^{(3)}, B_{m}^{(4)}$ are determined from the recurrence relations available on $\mathrm{E}$ resource (www. icmm.ru/compcoeff) and can be calculated using the suggested options. For this purposes, it is necessary to input the number of terms of a series $m$, Poisson's ratio $v$, the number of harmonics $k$ and the desired $\alpha$, which can be either complex or real.

Substituting (35) into (33), we obtain partial solutions $v_{0}^{(1)}, v_{0}^{(2)}, v_{0}^{(3)}, v_{0}^{(4)}$ for the function $v_{0}$

$$
\begin{aligned}
& v_{0}^{(1)}(x)=\frac{\sqrt{x(1-x)}}{[(\alpha-1) S-2]\left(\alpha+\alpha^{2}\right)} \sum_{m=0}^{\infty}\left[P_{m}^{(1)} x^{m}\right], \\
& v_{0}^{(2)}(x)=\frac{\sqrt{x(1-x)}}{[(\alpha-1) S-2]\left(\alpha+\alpha^{2}\right)} \sum_{m=0}^{\infty}\left[P_{m}^{(2)} x^{m}\right], \\
& v_{0}^{(3)}(x)=\frac{\sqrt{x(1-x)}}{[(\alpha-1) S-2]\left(\alpha+\alpha^{2}\right)}\left\{\frac{(1+S)}{x}+\sum_{m=0}^{\infty}\left[\left(P_{m}^{(3)}+D_{m}^{(3)} \ln (x)\right) x^{m}\right]\right\}, \\
& v_{0}^{(4)}(x)=\frac{\sqrt{x(1-x)}}{[(\alpha-1) S-2]\left(\alpha+\alpha^{2}\right)}\left\{\sum_{m=0}^{\infty}\left[\left(P_{m}^{(4)}+D_{m}^{(4)} \cdot \ln (x)\right) x^{(m-1)}\right]\right\},
\end{aligned}
$$

where the coefficients $P_{m}^{(1)}, P_{m}^{(2)}, P_{m}^{(3)}, P_{m}^{(4)}, D_{m}^{(3)}, D_{m}^{(4)}$ are available on E-resource (www. icmm.ru/compcoeff) and can be calculated ibidem.

A general solution for $u_{0}$ and $v_{0}$ can be written as

$$
\begin{aligned}
& u_{0}(x)=C_{1} \cdot u_{0}^{(1)}(x)+C_{2} \cdot u_{0}^{(2)}(x)+C_{3} \cdot u_{0}^{(3)}(x)+C_{4} \cdot u_{0}^{(4)}(x), \\
& v_{0}(x)=C_{1} \cdot v_{0}^{(1)}(x)+C_{2} \cdot v_{0}^{(2)}(x)+C_{3} \cdot v_{0}^{(3)}(x)+C_{4} \cdot v_{0}^{(4)}(x)
\end{aligned}
$$

where $C_{1}, C_{2}, C_{3}, C_{4}$ are the constants defined by the prescribed combination of boundary conditions (3) - (5).

\section{CONSTRUCTION OF PARTIAL SOLUTION FOR NONZEROTH HARMONICS OF THE FOURIER SERIES}

he construction of partial solutions to Eqns. (10)-(12) involves some transformations [21], which yield a system of two differential equations with respect to $w_{k}, v_{k}$

$$
\begin{aligned}
& f_{4}(x) \frac{d^{4} w_{k}(x)}{d x^{4}}+f_{3}(x) \frac{d^{3} w_{k}(x)}{d x^{3}}+f_{2}(x) \frac{d^{2} w_{k}(x)}{d x^{2}}+f_{1}(x) \frac{d w_{k}(x)}{d x}+f_{0}(x) w_{k}(x)=0 \\
& \psi_{2}(x) \frac{d^{2} v_{k}(x)}{d x^{2}}+\psi_{0}(x) v_{k}(x)=\phi_{3}(x) \frac{d^{3} w_{k}(x)}{d x^{3}}+\phi_{2}(x) \frac{d^{2} w_{k}(x)}{d x^{2}}+\phi_{1}(x) \frac{d w_{k}(x)}{d x}+\phi_{0}(x) w_{k}(x)
\end{aligned}
$$

where $f_{0}, f_{1}, f_{2}, f_{3}, f_{4}, \psi_{0}, \psi_{2}, \phi_{0}, \phi_{1}, \phi_{2}, \phi_{3}$ are given by

$$
\begin{aligned}
& f_{0}(x)=\frac{1}{2} x \alpha(\alpha+1)(x-1)\left[2 x(\alpha+3)(\alpha-2)(x-1)+k^{2}-1\right]+\frac{1}{16}(k-1)^{2}(k+1)^{2} \\
& f_{0}(x)=\frac{1}{2} x \alpha(\alpha+1)(x-1)\left[2 x(\alpha+3)(\alpha-2)(x-1)+k^{2}-1\right]+\frac{1}{16}(k-1)^{2}(k+1)^{2} \\
& f_{1}(x)=x(1-x)(2 x-1)\left[4 x\left(\alpha^{2}+\alpha-3\right)(x-1)+\frac{1}{2} k^{2}-\frac{1}{2}\right]
\end{aligned}
$$




$$
\begin{aligned}
& f_{2}(x)=-\frac{1}{2} x^{2}(x-1)^{2}\left[4 x\left(\alpha^{2}+\alpha-18\right)(x-1)+k^{2}-13\right] \\
& f_{3}(x)=6 x^{3}(x-1)^{3}(2 x-1) \quad f_{4}(x)=x^{4}(x-1)^{4} \\
& \psi_{0}(x)=x \alpha(\alpha+1)(x-1)+\frac{1}{4}\left(1-k^{2}\right) ; \psi_{2}(x)=x^{2}(x-1)^{2} \\
& \phi_{0}(x)=-\frac{1}{2} x\left[4 x \alpha(\alpha+1)(x-1)-k^{2}+1\right] \frac{(2 x-1)}{k} \\
& \phi_{1}(x)=\frac{1}{2} x\left[4 x\left(\alpha^{2}+\alpha-4\right)(x-1)-k^{2}+1\right] \frac{(x-1)}{k} \\
& \phi_{2}(x)=-5 x^{2}(2 x-1) \frac{(x-1)^{2}}{k} \phi_{3}(x)=2 x^{3} \frac{(x-1)^{3}}{k}
\end{aligned}
$$

Moreover, the results of these transformations is a set of equations, which relate the function $u_{k}$ to the functions $w_{k}, v_{k}$ and their derivatives

$$
\begin{aligned}
& u_{k}(x)=\frac{\sqrt{x(1-x)}}{2 x(x-1) k(S \alpha+2 S+2)}\left\{4 x^{2}(x-1) \frac{d^{2} w_{k}(x)}{d x^{2}}+4 x(2 x-1) \frac{d w_{k}(x)}{d x}-\right. \\
& \left.-\left[4 \alpha x(x-1)(1+\alpha)+k^{2}(S+1)+1\right] w_{k}(x)-\left[2 k S x(x-1) \frac{d v_{k}(x)}{d x}+k(2 x-1)(S+2) v_{k}(x)\right]\right\}
\end{aligned}
$$

Eqn. (38) is independent of (39) and represents a linear differential equation of the fourth order with respect to the function $w_{k}$. Eqn. (39) can be considered as a differential equation of the second order with respect to the function $v_{k}$ with right -hand side depending on $w_{k}$. Such a peculiarity of the differential Eqns. (28) and the obtained relation (30) allows us to determine the sequence of constructing partial solutions for functions $w_{k}, v_{k}, u_{k}$. The hang of this sequence can be outlines as follows. First, from the solution of Eqn. (38) we obtain four particular solutions $w_{k}^{(1)}, w_{k}^{(2)}, w_{k}^{(3)}, w_{k}^{(4)}$, written as

$$
\begin{aligned}
& w_{k}^{(1)}(x)=\sum_{m=0}^{\infty}\left[A_{m}^{(1)} x^{\left(m+\frac{k+1}{2}\right)}\right], \quad w_{k}^{(2)}(x)=\sum_{m=0}^{\infty}\left[A_{m}^{(2)} x^{\left(m+\frac{k-1}{2}\right)}\right], \\
& w_{k}^{(3)}(x)=\sum_{m=0}^{\infty}\left[\left(A_{m}^{(3)}+B_{m}^{(3)} \ln (x)\right) x^{\left(m-\frac{k-1}{2}\right)}\right], \quad w_{k}^{(4)}(x)=\sum_{m=0}^{\infty}\left[\left(A_{m}^{(4)}+B_{m}^{(4)} \ln (x)\right) x^{\left(m-\frac{k+1}{2}\right)}\right]
\end{aligned}
$$

where the coefficients $A_{m}^{(1)}, A_{m}^{(2)}, A_{m}^{(3)}, A_{m}^{(4)}, B_{m}^{(3)}, B_{m}^{(4)}$ can be determined and calculated E-resource (www. icmm.ru/compcoeff).

Then substituting the obtained particular solutions $w_{k}^{(1)}, w_{k}^{(2)}, w_{k}^{(3)}, w_{k}^{(4)}$ into the right-hand side of Eqn. (28. b) and solving it as an inhomogeneous equation we arrive at four partial solutions $v_{k}^{(1)}, v_{k}^{(2)}, v_{k}^{(3)}, v_{k}^{(4)}$, which can be written as

$$
\begin{aligned}
& v_{k}^{(1)}(x)=\sum_{m=0}^{\infty}\left[P_{m}^{(1)} x^{\left(m+\frac{k+1}{2}\right)}\right], \quad v_{k}^{(2)}(x)=\sum_{m=0}^{\infty}\left[P_{m}^{(2)} x^{\left(m+\frac{k-1}{2}\right)}\right] \\
& v_{k}^{(3)}(x)=\sum_{m=0}^{\infty}\left\{\left[P_{m}^{(3)}+D_{m}^{(3)} \ln (x)\right] x^{\left(m-\frac{k-1}{2}\right)}\right\}
\end{aligned}
$$




$$
v_{k}^{(4)}(x)=\sum_{m=0}^{\infty}\left\{\left[P_{m}^{(4)}+D_{m}^{(4)} \ln (x)\right] x^{\left(m-\frac{k+1}{2}\right)}\right\}
$$

where the coefficients $P_{m}^{(1)}, P_{m}^{(2)}, P_{m}^{(3)}, P_{m}^{(4)}, D_{m}^{(3)}, D_{m}^{(4)}$ can be determined and calculated E-resource (www. icmm.ru/compcoeff).

Then, solving Eqns. (39) as a homogeneous one, we find two more partial solutions $v_{k}^{(5)}, v_{k}^{(6)}$. The form of this differential equation indicates that the point $x=0$ is a regular singular point. The construction of partial solutions in the form of the generalized power series is accomplished in the framework of the proposed approach. The obtained partial solutions are expressed as

$$
v_{k}^{(5)}(x)=\sum_{m=0}^{\infty}\left[P_{m}^{(5)} x^{\left(m+\frac{k+1}{2}\right)}\right], v_{k}^{(6)}(x)=\sum_{m=0}^{\infty}\left[\left(P_{m}^{(6)}+D_{m}^{(6)} \ln (x)\right) x^{\left(m+\frac{k-1}{2}\right)}\right]
$$

where the coefficients $P_{m}^{(5)}, P_{m}^{(6)}, D_{m}^{(6)}$ can be determined and calculated E-resource (www. icmm.ru/compcoeff).

At the next stage, we use the partial solutions $w_{k}^{(1)}, w_{k}^{(2)}, w_{k}^{(3)}, w_{k}^{(4)}, v_{k}^{(1)}, v_{k}^{(2)}, v_{k}^{(3)}, v_{k}^{(4)}, v_{k}^{(5)}, v_{k}^{(6)}$ and the obtained relation (40) to derive six partial solutions $u_{k}^{(1)}, u_{k}^{(2)}, u_{k}^{(3)}, u_{k}^{(4)}, u_{k}^{(5)}, u_{k}^{(6)}$, which are expressed as

$$
\begin{aligned}
& u_{k}^{(1)}=\frac{2 \sqrt{x(1-x)}}{k x(x-1)(S(\alpha+2)+2)} \sum_{m=0}^{\infty}\left[E_{m}^{(1)} x^{\left(m+\frac{k+1}{2}\right)}\right] \\
& u_{k}^{(2)}=\frac{2 \sqrt{x(1-x)}}{k x(x-1)(S(\alpha+2)+2)} \sum_{m=0}^{\infty}\left[E_{m}^{(2)} x^{\left(m+\frac{k-1}{2}\right)}\right] \\
& u_{k}^{(3)}=\frac{2 \sqrt{x(1-x)}}{k x(x-1)(S(\alpha+2)+2)} \sum_{m=0}^{\infty}\left[\left(E_{m}^{(3)}+G_{m}^{(3)} \ln (x)\right) x^{\left(m-\frac{k-1}{2}\right)}\right] \\
& u_{k}^{(4)}=\frac{2 \sqrt{x(1-x)}}{k x(x-1)(S(\alpha+2)+2)} \sum_{m=0}^{\infty}\left[\left(E_{m}^{(4)}+G_{m}^{(4)} \ln (x)\right) x^{\left(m-\frac{k+1}{2}\right)}\right] \\
& u_{k}^{(5)}=\frac{\sqrt{x(1-x)}}{k x(x-1)(S(\alpha+2)+2)} \sum_{m=0}^{\infty}\left[E_{m}^{(5)} x^{\left(m+\frac{k+1}{2}\right)}\right] \\
& u_{k}^{(6)}=\frac{\sqrt{x(1-x)}}{k x(x-1)(S(\alpha+2)+2)} \sum_{m=0}^{\infty}\left[\left(E_{m}^{(6)}+G_{m}^{(6)} \ln (x)\right) x^{\left(m-\frac{k-1}{2}\right)}\right]
\end{aligned}
$$

where the coefficients $E_{m}^{(1)}, E_{m}^{(2)}, E_{m}^{(3)}, E_{m}^{(4)}, E_{m}^{(5)}, E_{m}^{(6)}, G_{m}^{(3)}, G_{m}^{(4)}, G_{m}^{(6)}$ for any value of $m \geq 0$ can be determined and calculated E-resource (www. icmm.ru/compcoeff).

The general solutions for $u_{k}, v_{k}, w_{k}$ are given by

$$
\begin{aligned}
& u_{k}(x)=C_{1} \cdot u_{k}^{(1)}(x)+C_{2} \cdot u_{k}^{(2)}(x)+C_{3} \cdot u_{k}^{(3)}(x)+C_{4} \cdot u_{k}^{(4)}(x)+C_{5} \cdot u_{k}^{(5)}(x)+C_{6} \cdot u_{k}^{(6)}(x) \\
& v_{k}(x)=C_{1} \cdot v_{k}^{(1)}(x)+C_{2} \cdot v_{k}^{(2)}(x)+C_{3} \cdot v_{k}^{(3)}(x)+C_{4} \cdot v_{k}^{(4)}(x)+C_{5} \cdot v_{k}^{(5)}(x)+C_{6} \cdot v_{k}^{(6)}(x) \\
& v_{k}(x)=C_{1} \cdot v_{k}^{(1)}(x)+C_{2} \cdot v_{k}^{(2)}(x)+C_{3} \cdot v_{k}^{(3)}(x)+C_{4} \cdot v_{k}^{(4)}(x)+C_{5} \cdot v_{k}^{(5)}(x)+C_{6} \cdot v_{k}^{(6)}(x) \\
& w_{k}(x)=C_{1} \cdot w_{k}^{(1)}(x)+C_{2} \cdot w_{k}^{(2)}(x)+C_{3} \cdot w_{k}^{(3)}(x)+C_{4} \cdot w_{k}^{(4)}(x)
\end{aligned}
$$


where $C_{1}, C_{2}, C_{3}, C_{4}, C_{5}, C_{6}$ are the constants defined by the prescribed combination of boundary conditions (3) - ( 5).

\section{EXAMPLE OF CALCULATION OF CHARACTERISTIC INDICES FOR CONICAL BODIES}

7 he general solutions obtained for $k=0$ and $k \geq 1$ under the prescribed combination of the boundary conditions were used to construct a homogeneous system of algebraic equations with respect to constants $C_{i}$ for the examined conical body. The coefficients of this system of equations depend on the vertex angles of conical bodies, elastic characteristics of the materials and characteristic index $\alpha$. The indices $\alpha$, defining the character of stress singularity at the vertices of conical bodies are calculated from the condition of the existence of non-zero solution to the system of linear algebraic equations.

\section{Solid cone}

Let us consider a solid cone $\left(0 \leq r \leq \infty, 0 \leq \varphi \leq, 0 \leq \theta \leq \theta_{0}\right)$. For this case, in general solutions (32), (37), (45) it is necessary to retain terms with particular solutions (15) at $x=0$ (or $\theta=0$ ).

Subject to this condition the solutions take the following form:

axisymmetric rotation $(k=0)$

$$
w_{0}(x)=C_{0}^{(1)} \cdot w_{0}^{(1)}(x)
$$

axisymmetric deformation $(k=0)$

$$
\begin{aligned}
& u_{0}(x)=C_{0}^{(1)} \cdot u_{0}^{(1)}(x)+C_{0}^{(2)} \cdot u_{0}^{(2)}(x), \\
& v_{0}(x)=C_{0}^{(1)} \cdot v_{0}^{(1)}(x)+C_{0}^{(2)} \cdot v_{0}^{(2)}(x)
\end{aligned}
$$

non-axisymmetric deformation $(k>0)$

$$
\begin{aligned}
& u_{k}(x)=C_{k}^{(1)} \cdot u_{k}^{(1)}(x)+C_{k}^{(2)} \cdot u_{k}^{(2)}(x)+C_{k}^{(5)} \cdot u_{k}^{(5)}(x) \\
& v_{k}(x)=C_{k}^{(1)} \cdot v_{k}^{(1)}(x)+C_{k}^{(2)} \cdot v_{k}^{(2)}(x)+C_{k}^{(5)} \cdot v_{k}^{(5)}(x) \\
& w_{k}(x)=C_{k}^{(1)} \cdot w_{k}^{(1)}(x)+C_{k}^{(2)} \cdot w_{k}^{(2)}(x)
\end{aligned}
$$

The substitution of these solutions in one of the boundary conditions (3)-(5) at $\theta=\theta_{0}$ yields a system of linear homogeneous algebraic equations for the unknown quantities, $C_{0}^{(1)}, C_{0}^{(2)}, C_{k}^{(1)}, C_{k}^{(2)}, C_{k}^{(5)}$, which have the following representation:

axisymmetric rotation $(k=0)$

$$
C_{0}^{(1)} \cdot \Phi_{0}^{(1,1)}=0
$$

axisymmetric deformation $(k=0)$

$$
\begin{aligned}
& C_{0}^{(1)} \cdot \Psi_{0}^{(1,1)}+C_{0}^{(2)} \cdot \Psi_{0}^{(1,2)}=0 \\
& C_{0}^{(1)} \cdot \Psi_{0}^{(2,1)}+C_{0}^{(2)} \cdot \Psi_{0}^{(2,2)}=0 .
\end{aligned}
$$

non-axisymmetric deformation $(k>0)$ 


$$
\begin{aligned}
& C_{k}^{(1)} \cdot \Psi_{k}^{(1,1)}+C_{k}^{(2)} \cdot \Psi_{k}^{(1,2)}+C_{k}^{(5)} \cdot \Psi_{k}^{(1,5)}=0, \\
& C_{k}^{(1)} \cdot \Psi_{k}^{(2,1)}+C_{k}^{(2)} \cdot \Psi_{k}^{(2,2)}+C_{k}^{(5)} \cdot \Psi_{k}^{(2,5)}=0, \\
& C_{k}^{(1)} \cdot \Psi_{k}^{(5,1)}+C_{k}^{(2)} \cdot \Psi_{k}^{(5,2)}+C_{k}^{(5)} \cdot \Psi_{k}^{(5,5)}=0 .
\end{aligned}
$$

A particular form of the coefficients $\Phi_{0}^{(1,1)}, \Psi_{0}^{(1,1)}, \Psi_{0}^{(1,2)}, \Psi_{0}^{(2,1)}, \Psi_{0}^{(2,2)}, \Psi_{k}^{(1,1)}, \ldots \Psi_{k}^{(5,5)}$ will be specified by the selected boundary conditions, but in any case these coefficients will depend on the finite series determining the form of particular solutions $w_{0}^{(1)}, u_{0}^{(1)}, v_{0}^{(1)}, u_{0}^{(2)}, v_{0}^{(2)}, u_{k}^{(1)}, v_{k}^{(1)}, w_{k}^{(1)}, u_{k}^{(2)}, v_{k}^{(2)}, w_{k}^{(2)}, u_{k}^{(5)}, v_{k}^{(5)}$ the cone angle, the elastic characteristics of the material and the sought-for characteristic index $\alpha$.

The condition for the existence of nontrivial solutions to the systems of the algebraic Eqns. (49) - (51) are written as

$$
\begin{aligned}
& \Phi_{0}^{(1,1)}=0 \\
& \operatorname{det}\left\{\begin{array}{ll}
\Psi_{0}^{(1,1)} & \Psi_{0}^{(1,2)} \\
\Psi_{0}^{(2,1)} & \Psi_{0}^{(2,2)}
\end{array}\right\}=0 \\
& \operatorname{det}\left\{\begin{array}{lll}
\Psi_{0}^{(1,1)} & \Psi_{0}^{(1,2)} & \Psi_{0}^{(1,5)} \\
\Psi_{0}^{(2,1)} & \Psi_{0}^{(2,2)} & \Psi_{0}^{(2,5)} \\
\Psi_{0}^{(5,1)} & \Psi_{0}^{(5,2)} & \Psi_{0}^{(5,5)}
\end{array}\right\}=0
\end{aligned}
$$

From these relations we obtain the values of the characteristic indices $\alpha$ for each of the examined cases (axisymmetric rotation, axisymmetric deformation and non-axisymmetric deformation).

When implementing numerically the algorithm for evaluation of the characteristic indices one should bear in mind that the number of terms in the series of constructed particular solutions (20), (30), (35), (36), (41) - (44) is selected in such a way that a subsequent increase in their number do not change the values of the characteristic indices in the third decimal place. It should be noted that the most "unfavorable" cases required the retention of 300-400 series terms. Fig. 2 presents the values of $\operatorname{Re} \alpha_{n}<1$, determining singular solutions for a solid cone under the boundary conditions expressed in terms of displacements and stresses. The values given on the graphs are identical to the data presented in [19].
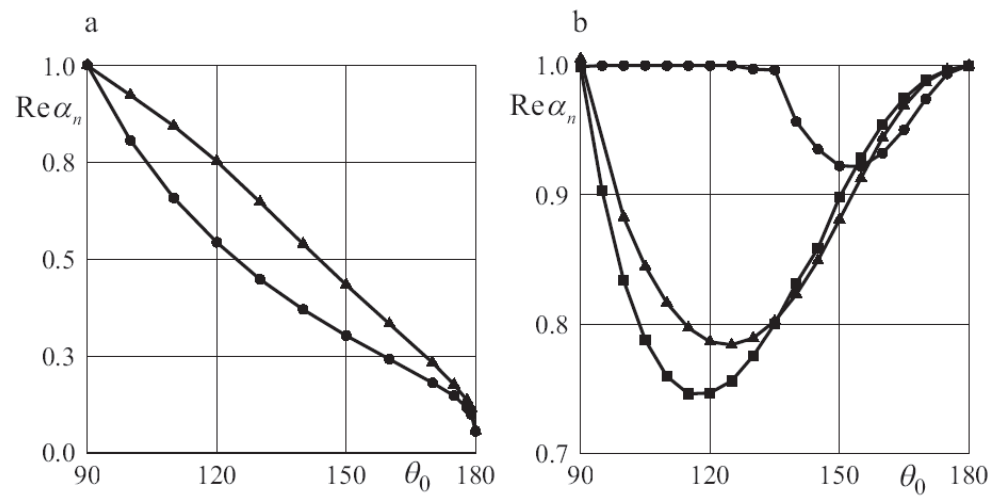

Figure 2: $\operatorname{Re} \alpha_{n}<1$ as a function of the vertex angle of the solid cone with boundary conditions on the lateral surface for displacements (a) and stresses (b) $(\boldsymbol{\Lambda}-k=0, \bullet-k=1, \boldsymbol{\square}-k=2)$.

It should be noted that the singular solutions for a solid cone under the boundary conditions in terms of stresses take place for the zeroth, first and second harmonics of the Fourier series and under the boundary conditions in terms of displacements- for the zeroth and first harmonics of the Fourier series. 
All results in this paper were obtained for a material with Poisson's ratio $v=0.3$.

Fig. 3 displays new data on the character of stress singularity at the vertex of the solid cone for the perfect-slip boundary conditions at the lateral surface. Here singular solutions occur at the zeroth first and second harmonics of the Fourier series and at the angle $\theta_{0}$ less than $180^{\circ}$.

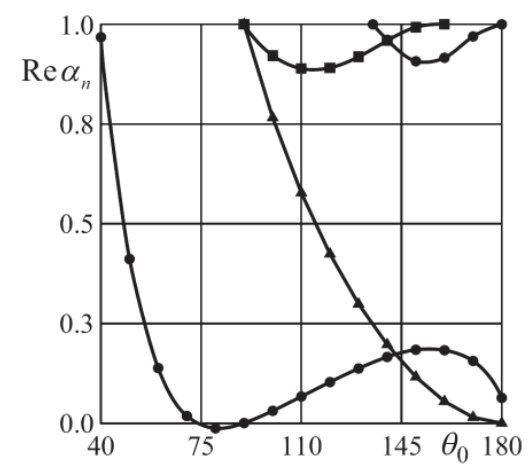

Figure 3: $\operatorname{Re} \alpha_{n}<1$ as a function of the vertex angle of the solid cone under the perfect slip boundary conditions on the lateral surface $(\boldsymbol{\Lambda}-k=0, \bullet-k=1, \boldsymbol{\square}-k=2)$.
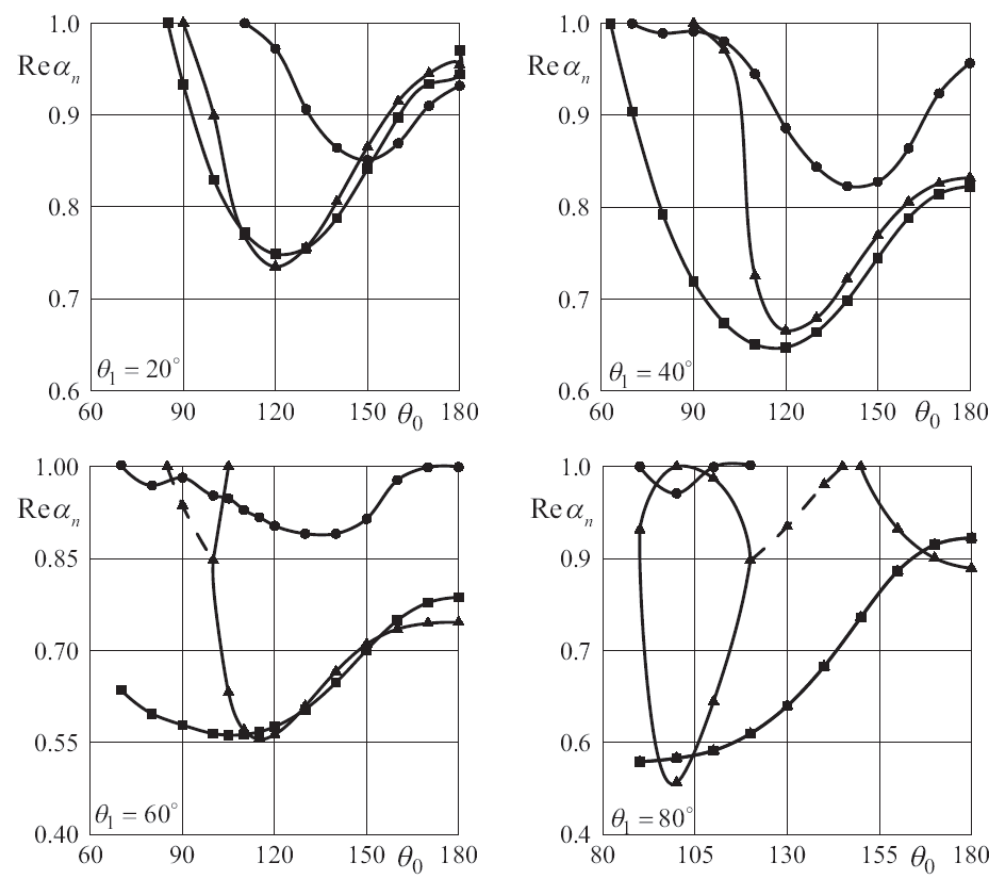

Figure 4: Variation $\operatorname{Re} \alpha_{n}<1$ with the angle $\theta_{0}$ at fixed values of the angle $\theta_{1}$ of the hollow cone under zero-stress boundary conditions on the lateral surface $(\boldsymbol{\Delta}-k=0, \bullet-k=1, \mathbf{\square}-k=2)$.

The algorithm for evaluation of the characteristic indices described above was applied to a hollow cone with two conical boundary surfaces $\theta=\theta_{0}$ and $\theta=\theta_{1}$ subject to different homogeneous boundary conditions (13) - (15). Note that in search for a solution to this problem we had to use a full set of particular solutions $\left(w_{0}^{(1)}, w_{0}^{(2)}, u_{0}^{(1)}, v_{0}^{(1)}, u_{0}^{(2)}, v_{0}^{(2)}, u_{0}^{(3)}, v_{0}^{(3)}, u_{0}^{(4)}, v_{0}^{(4)}, u_{k}^{(1)}, v_{k}^{(1)}, w_{k}^{(1)}, u_{k}^{(1)}, v_{k}^{(1)}, w_{k}^{(1)}, u_{k}^{(2)}, v_{k}^{(2)}, w_{k}^{(2)}, u_{k}^{(3)}, v_{k}^{(3)}, w_{k}^{(3)}, u_{k}^{(4)}, v_{k}^{(4)}, w_{k}^{(4)}\right.$, $u_{k}^{(5)}, v_{k}^{(5)}, u_{k}^{(6)}, v_{k}^{(6)}$ ) defined by relations (20), (30), (35), (36), (41) - (44). Since the number of particular solutions doubled, the order of determinants (52) - (54) doubled, too. 
Fig. 4 shows the dependence of the eigenvalues $\operatorname{Re} \alpha_{n}<1$ on the cone angle formed by the external surface $\theta_{0}$ at different values of the internal cone angle $\theta_{1}$. The conical surfaces meet zero boundary conditions for stresses. Here the solid line corresponds to real eigenvalues and the dashed line - to complex eigenvalues.

In Fig. 5, the dependence of eigenvalues $\operatorname{Re} \alpha_{n}<1$ on $\theta_{0}$ is plotted for two values of the internal cone angle for the case when the conical surfaces meet zero boundary conditions for displacements.

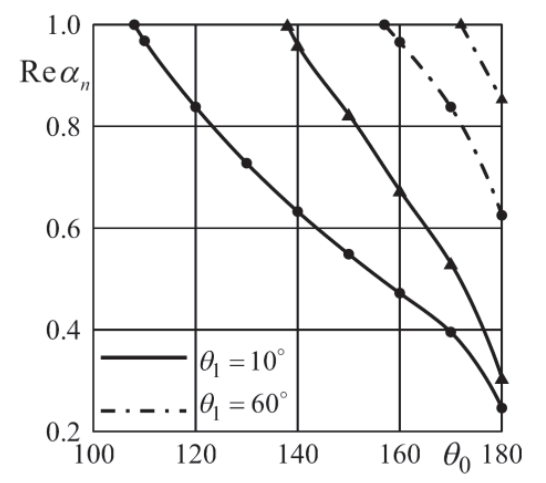

Figure 5: Variation $\operatorname{Re} \alpha_{n}<1$ with the angle $\theta_{0}$ at fixed values of the angle $\theta_{1}(\boldsymbol{\Delta}-k=0, \bullet-k=1)$ of the hollow cone under zerostress boundary conditions on the lateral surface.

For a hollow cone different combinations of the boundary conditions can be realized at the internal and external conical surfaces. We have considered two variants. In the first case, the internal surface meets the zero-stress boundary conditions and the external surface - the condition of zero displacements. In the second case the condition of zero displacements is prescribed on the internal surface and on the internal surface - the condition of zero stresses.

The variation of the stress singularity index $\operatorname{Re} \alpha_{n}<1$ with the cone angle $\theta_{0}$ formed by the external surface at different values of the internal cone angle is shown in Fig. 6. The eigenvalues, at which singular stresses occur, are observed at the values of $\theta_{0}$ higher than $80^{\circ}$.
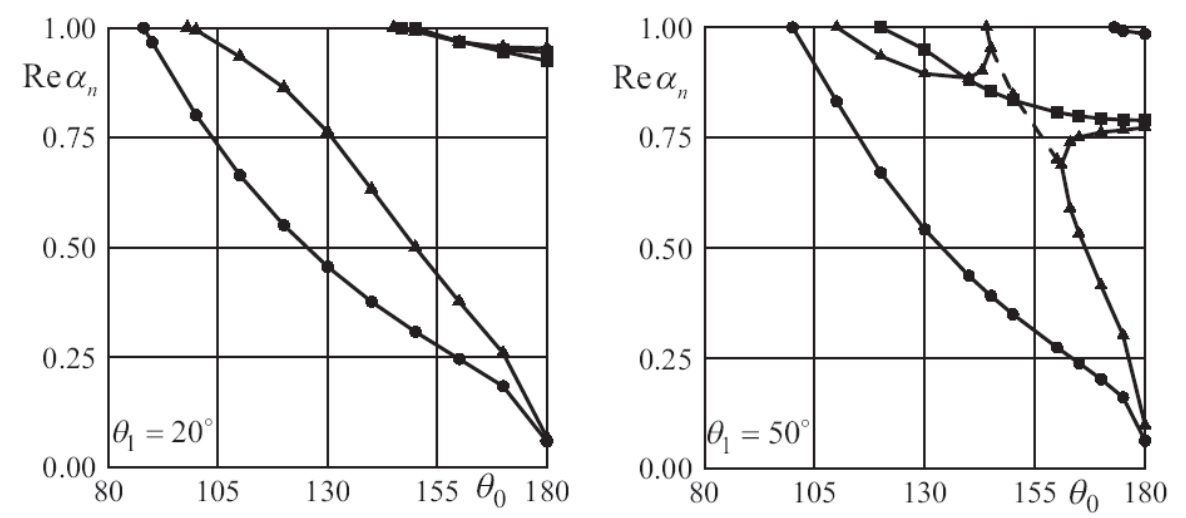

Figure 6: $\operatorname{Re} \alpha_{n}<1$ as a function of $\theta_{0}$ for different values of the cone angle $\theta_{1}$ at zero stresses on the internal surface and zero displacements on the external lateral surfaces $(\boldsymbol{\Delta}-k=0, \bullet-k=1, \boldsymbol{\square}-k=2)$.

For the second variant of the boundary conditions the dependence of the values of $\operatorname{Re} \alpha_{n}<1$ on $\theta_{0}$ is shown in Fig. 7.

\section{Composite cone}

In the case of a composite cone the algorithm for computation of characteristic indices presented in subparagraph "Solid cone" can be applied to different versions of a composite cone. First we consider a composite cone with a single boundary conical surface $\theta=\theta_{0}$ and contact boundary $\theta=\theta_{2}$. A solution to this problem is found by using regular particular solutions for the internal sub-domain $\left(0 \leq r<\infty, \theta_{1} \leq \theta \leq \theta_{2}, 0 \leq \varphi \leq 2 \pi\right)$ and non-regular particular solutions defined by relations ((20), (30), (35), (36), (41) - (44)) for the external sub-domain $\left(0 \leq r<\infty, \theta_{2} \leq \theta \leq \theta_{0}\right.$, 
$0 \leq \varphi \leq 2 \pi)$. As second case we consider a composite cone with two boundary conical surfaces, and contact boundary. In this case, it is necessary to use a full set of particular $\left(w_{0}^{(1)}, w_{0}^{(2)}, u_{0}^{(1)}, v_{0}^{(1)}, u_{0}^{(2)}, v_{0}^{(2)}, u_{0}^{(3)}, v_{0}^{(3)}, u_{0}^{(4)}, v_{0}^{(4)}, u_{k}^{(1)}, v_{k}^{(1)}, w_{k}^{(1)}, u_{k}^{(1)}, v_{k}^{(1)}, w_{k}^{(1)}, u_{k}^{(2)}, v_{k}^{(2)}, w_{k}^{(2)}, u_{k}^{(3)}, v_{k}^{(3)}, w_{k}^{(3)}, u_{k}^{(4)}, v_{k}^{(4)}, w_{k}^{(4)}\right.$, $u_{k}^{(5)}, v_{k}^{(5)}, u_{k}^{(6)}, v_{k}^{(6)}$ ) defined by relations ((20), (30), (35), (36), (41)-(44)) both for the internal and external sub-domains.
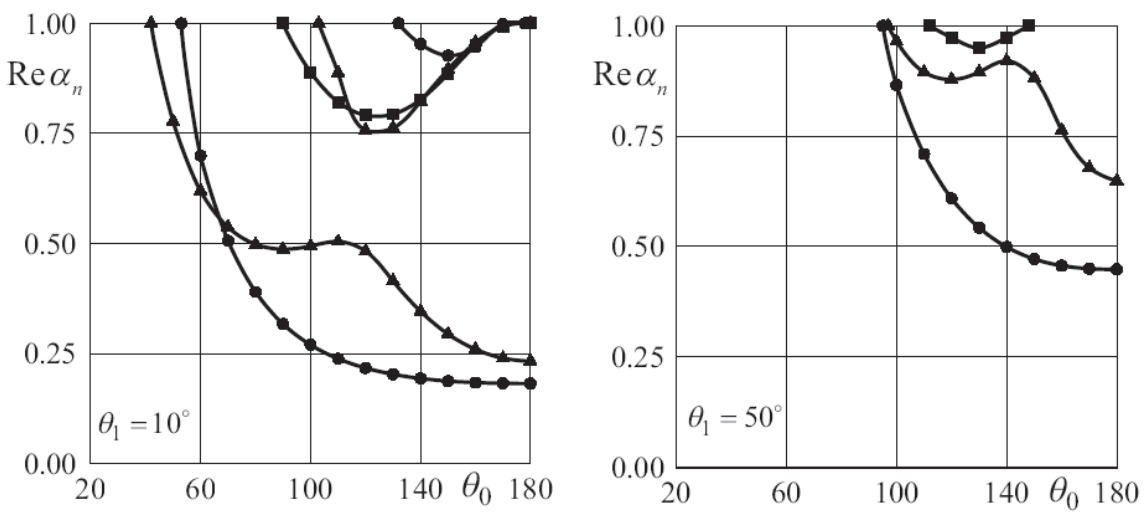

Figure 7: $\operatorname{Re} \alpha_{n}<1$ as a function of $\theta_{0}$ for different values of the cone angle $\theta_{1}$ of the hollow cone at zero displacements on the internal surface and zero stresses on the external lateral surfaces $(\boldsymbol{\Delta}-k=0, \bullet-k=1, \boldsymbol{\square}-k=2)$.
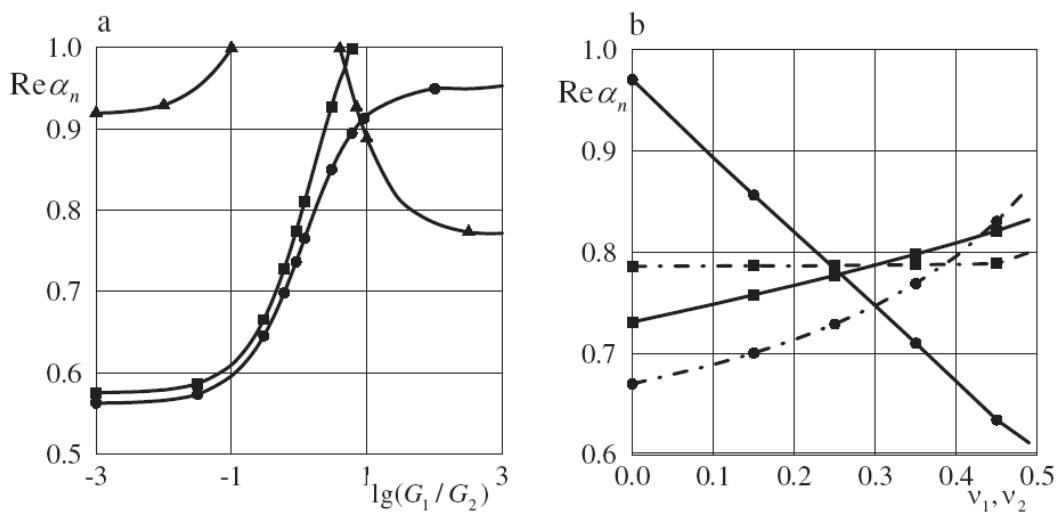

Figure 8: $\operatorname{Re} \alpha_{n}<1$ at different values of $\log \left(G_{1} / G_{2}\right)$ for $\theta_{2}=60^{\circ}, \theta_{0}=120^{\circ}, v_{1}=v_{2}=0.3$ (a). Re $\alpha_{n}<1$ at different values of Poison`s coefficient for $v_{1}, v_{2}$ for $\theta_{2}=60^{\circ}, \theta_{0}=120^{\circ}$ (b). $(\boldsymbol{\Delta}-k=0, \bullet-k=1, \boldsymbol{\square}-k=2)$.

As an example, let us consider a composite cone with zero stress conditions on the external lateral boundary surface and perfect contact conditions on the boundary between different cone parts made of dissimilar materials. Fig. 8a shows variation $\operatorname{Re} \alpha_{n}<1$ of with the ratio between shear modules of different materials $G_{1} / G_{2}$. The dependence of eigenvalues on Poisson's ratio for $G_{1} / G_{2}=1$ is given in Fig. 8b. Solid lines denote eigenvalues for $v_{1}=0.3$ as a function of $v_{2}$ and dash and dot lines denote eigenvalues for $v_{2}=0.3$ as a function of $v_{1}$.

Of particular interest is a composite cone with the internal singular point. In this case, two types of conditions can be prescribed at the boundary between two different materials $\theta=\theta_{2}$ (perfect bonding condition (8) or frictionless slip conditions (9). The stress singularity indices shown in Fig. 9 correspond to condition (8) for different shear modules ratios of different cone layers. It has been found that at the internal singular point the condition of stress singularity is realized at any ratio between shear modules of the inner and outer cones, except for the trivial case of $\theta_{2}=90^{\circ}$. 


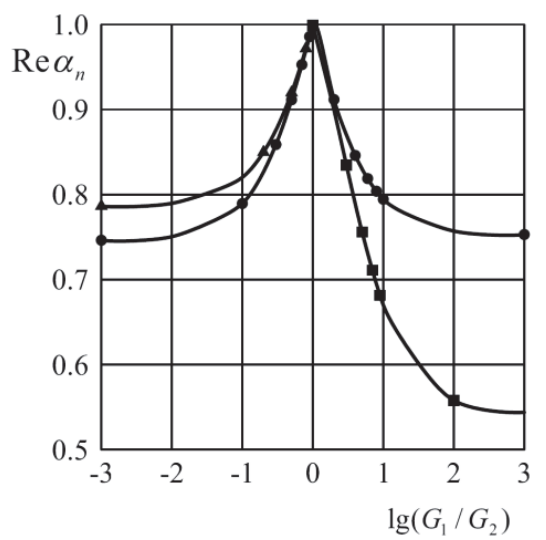

Figure 9: Variation of $\operatorname{Re} \alpha_{n}<1$ with $\log \left(G_{1} / G_{2}\right)$ in condition of perfect contact between two different materials at $\theta_{2}=60^{\circ}, v_{1}=v_{2}=0.3 \cdot(\mathbf{\Delta}-k=0, \bullet-k=1, \boldsymbol{\square}-k=2)$.

\section{CONCLUSION}

$\mathrm{W}$

e have considered the analytical method of constructing eigensolutions for circular cones. It has been shown that the proposed analytical relations can be used to construct solutions and to evaluate the character of stress singularity for different conical bodies (solid, composite) and different types of boundary conditions on the lateral surfaces and contact surface of dissimilar materials. The numerical results presented in this paper provide information about the character of singular stresses at the vertex of a solid cone for displacement, stress and combined boundary conditions and at the vertex of a hollow cone for different boundary conditions on the internal and external lateral surfaces.

The importance of the theoretical output of this research is that the obtained analytical solutions provide full information about singular solutions for different types of circular conical bodies made of isotropic elastic materials. All formulas and options for calculation, allowing readers to obtain independently numerical results for the problems considered in the paper, can be found on E-resource (www. icmm.ru/compcoeff).

The results of this work may have considerable practical implications in various fields. Recent advances in the development of computational methods extend the possibilities of the in-depth analysis of the stress state, so that present computations of real objects provide a great deal of information on the stresses in the vicinity of singular points, which are often the stress concentrator zones. A search for effective ways of reducing the level of stress concentration, for example, by changing the geometry in the vicinity of singular points leads to the conclusion that the variants close to optimal are related with the character of singular solutions [22]. Such conclusions have found commercial applications, for example, for the development of techniques allowing engineers to increase the strength of adhesive or glue [23-25]. In this respect, the results presented in the paper can be used to find an appropriate variant of reducing the level of stress concentration in the vicinity of non-smooth points on the surface of three-dimensional elastic bodies. The obtained information has its own practical value since it allows us to estimate the character of stress behavior at the vertices of different conical natural objects and engineering structures. Moreover, the available data on the indices of stress singularity open up new possibilities for construction of singular finite elements [22].

\section{REFERENCES}

[1] Kondratiev, V.A. (1967). Boundary value problems for elliptic equations in the regions with conical and angular points. Transactions of the Moscow Mathematical Society 16, pp. 209-292.

[2] Sinclair, G.B. (2004). Stress singularities in classical elasticity - I: Removal, interpretation, and analysis. Applied Mechanics Reviews 57(4), pp. 251-297.

[3] Sinclair, G.B. (2004). Stress singularities in classical elasticity - II: Asymptotic identification. Applied Mechanics Reviews 57(5), pp. 385-439. 
[4] Mikhailov, S.E. (1979). Stress singularity in the vicinity of a rib of inhomogeneous composite anisotropic body and some applications to composites. Proceedings of the Academy of Sciences of the USSR. Solid Mechanics. 5, pp. 103110.

[5] Apel, T., Mehrmann, V., Watkins, D. (2002). Structured eigenvalue methods for the computation of corner singularities in 3D anisotropic elastic structures. Computer Methods in Applied Mechanics and Engineering 191, pp. 4459-4473. DOI: 10.1016/S0045-7825(02)00390-0

[6] Babeshko, V.A., Glushkov, E.V., Glushkova, N.V., Lapina, O.N. (1991). Stress singularity in the vicinity of the vertex of elastic trihedral. Proceedings of the Russian Academy of Sciences 318(5), pp. 1113-1116.

[7] Dimitrov, A., Andra, H., Schnack, E. (2002). Singularities near three-dimensional corners in composite laminates. International Journal of Fracture 115, pp. 361-375. DOI: 10.1002/nme.230

[8] Matveenko, V.P., Nakaryakova, T.O., Sevodina, N.V., Shardakov, I.N. (2006). Investigation of Singularity of Stresses at the Apex of a Cone with an Elliptic Base. Doklady Physics 51(11), pp. 630-633.

DOI: $10.1134 /$ S1028335806110140.

[9] Matveenko, V.P., Nakaryakova, T.O., Sevodina, N.V., Shardakov, I.N. (2008). Stress singularity at the vertex of homogeneous and composite cones for different boundary conditions. Journal of Applied Mathematics and Mechanics 72, pp. 331-337. DOI: 10.1016/j.jappmathmech.2008.07.012.

[10] Korepanova, T.O., Matveenko, V.P., Sevodina, N.V. (2010). Numerical analysis of stress singularity at the vertex of a cone with unsmooth lateral surface. Computer Mechanics of continuum Mechanics 3(3), pp. 68-76.

[11] Korepanova, T.O., Matveenko, V.P., Sevodina, N.V. (2013). Numerical analysis of stress singularity at singular points of three-dimensional elastic bodies. Acta Mechanica 224, pp. 2045-2063. DOI 10.1007/s00707-013-0845-y.

[12] Savruk, M.P. and Shkaraev, S.V. (2001). Stress singularities for three-dimensional corners using the boundary integral equation method. Theoretical Applied Mechanics 36, pp. 263-275.

[13] Bazant, Z.P., Keer, L.M. (1974). Singularities of elastic stresses and of harmonic functions at conical notches or inclusions. International Journal of Solids and Structures 10(9), pp. 957-965.

[14] Beagles, A.E. and Sänding, A.M. (1991). Singularities of rotationally symmetric solutions of boundary value problems for the Lame equations. ZAMM. Journal of Applied Mathematics and Mechanics. 71(11), pp. 423-431. DOI: 10.1002/zamm.19910711102.

[15] Bernardi, C., Dauge, M., Maday, Y. (1999). Spectral Methods for Axisymmetric Domains. Series in Applied Mathematics 3.

[16] Parihar, K.S., Keer, L.M. (1978). Elastic stress singularities at conical inclusions. International Journal of Solids and Structures 14, pp. 261-263. DOI: 10.1016/0020-7683(78)90036-7.

[17] Picu, C.R. (1996). Stress singularities at vertices of conical inclusions with freely sliding interfaces, International Journal of Solids and Structures 33(17), pp. 2453-2457. DOI: 10.1016/0020-7683(95)00164-6.

[18] Somaranta, N., Ting, T.C.T. (1986). Three dimensional stress singularities at conical notches and inclusions in transversely isotropic materials, Journal of Applied Mechanics 53, pp. 89-96.

[19] Kozlov, V.A., Maz'ya, V.G., Rossmann, J. (2001). Spectral Problems Associated with Corner Singularities of Solutions to Elliptic Equations. Providence RI.N.J.: American Mathematical Society, ser. Mathematical Surveys and Monographs 85. DOI: http://dx.doi.org/10.1090/surv/085.

[20] Nowacki, W. (1970). Theory of Elasticity. PWN. Warsaw.

[21] Kamke, E. (1959). Differentialgleichungen: Lösungsmethoden und Lösungen. Gewöhnliche Differentialgleichungen, Leipzig.

[22] Matveyenko, V.P. and Borzenkov, S.M. (1996). Semianalytical singular element and its application to stress calculation and optimization. International Journal for Numerical Methods in Engineering 39(10), pp. 1659-1680.

DOI: 10.1002/(SICI)1097-0207(19960530)39

[23] He, D., Sawa, T., Karami, A. (2009). Stress analysis and strength evaluation of scarf adhesive joints with dissimilar adherends subjected to static tensile loadings, International Journal of Adhesion and Adhesives 3(8), pp. 1033-1044.

[24] Lang, T.P., Mallick, P.K. (1998). Effect of spew geometry on stresses in single lap adhesive joints. International Journal of Adhesion and Adhesives 18, pp. 167-177. DOI: 10.1016/S0143-7496(97)00056-0.

[25] Xu, L.R., Kuai, H., Sengupta, S. (2004). Dissimilar material joints with and without free-edge stress singularities: Part II. An integrated numerical analysis. Experimental mechanics 44(6), pp. 616-621. DOI: 10.1007/BF02428251. 